\title{
Principle investigations on polarization image sensors
}

\section{David Rebhan, Maik Rosenberger, Gunther Notni}

David Rebhan, Maik Rosenberger, Gunther Notni, "Principle investigations on polarization image sensors," Proc. SPIE 11144, Photonics and Education in Measurement Science 2019, 111440A (17 September 2019); doi:

$10.1117 / 12.2533590$

SPIE Event: Joint TC1 - TC2 International Symposium on Photonics and Education in Measurement Science 2019, 2019, Jena, Germany 


\title{
Principle investigations on polarization image sensors
}

\author{
David Rebhan ${ }^{1}$, Maik Rosenberger ${ }^{1}$, Gunther Notni ${ }^{1}$ \\ ${ }^{1}$ Technische Universität Ilmenau, Faculty of Mechanical Engineering, \\ Group for Quality Assurance and Industrial Image Processing, \\ Gustav-Kirchhoff-Platz 2, 98693 Ilmenau, Germany
}

\begin{abstract}
With the appearance of cameras containing newly developed polarized image sensors, which include an on-chip polarizer array placement, there is an upcoming need to characterize these cameras according to a uniform standard. These polarizer arrays consist of $2 \times 2$ matrices with four different angled polarizers, placed on top of each pixel. Therefore, this setup differs from the traditionally used multi camera and multi-polarizer setups. In terms of characterizing cameras, the EMVA 1288 standard already provides a widely established guideline on how to characterize monochrome and color digital cameras with linear photo response characteristics. The aim of this paper is to characterize a polarization camera, which takes advantage of these newly developed sensors, within the framework of the EMVA 1288 standard. Therefore, a measuring setup is constructed to be able to isolate the response characteristics of the individual pixels of the polarizer arrays. The measuring setup is built in accordance to the EMVA 1288 standard, thus consisting of a monochromatic light source, a measurement tube and a photodiode. In addition, a rotatable linear polarizing filter is being placed in front of the image sensor, to be able to control the polarization direction of the incident light. The results obtained will then be evaluated and interpreted, and proposals for an extension of the EMVA 1288 standard for the characterization of polarization cameras will be given, based on the experience gained throughout the characterization.
\end{abstract}

\section{INTRODUCTION}

Traditional systems for the detection of polarized light in an industrial application either consist of a multiple camera system with different angled polarizers or of a single camera with a mechanical driven polarizer. With the IMX250MZR (monochrome) and IMX250MYR (color) Sony released new sensors that have filter on chip (FOC) wire grid polarizers in front of each pixel in a 2x2 matrix pattern. Figure 1 (left) shows the principle structure of the IMX250MZR sensor. Each pixel has a size of $3.45 \mu \mathrm{m}$ and the sensor has an effective resolution of 5.07 Megapixels [1]. Due to the manufacturing process, the wire grid array is placed between the photodiodes and the on-chip glass and lenses, which improves the extinction ratio and the incident angle dependence [1]. Because of the four different angled linear polarizers of the matrix, it is possible to calculate the degree of linear polarization (DoLP) and the angle of linear polarization (AoLP) which are necessary for industrial applications such as scratch detection, particle inspection, distortion and shape detection [1]. Figure 1 (right) shows an image of a transparent ruler, taken with the IMX250MZR sensor. The DoLP and AoLP are transferred to the HSV color space. This makes it possible to see the internal stress of the material in a live image.
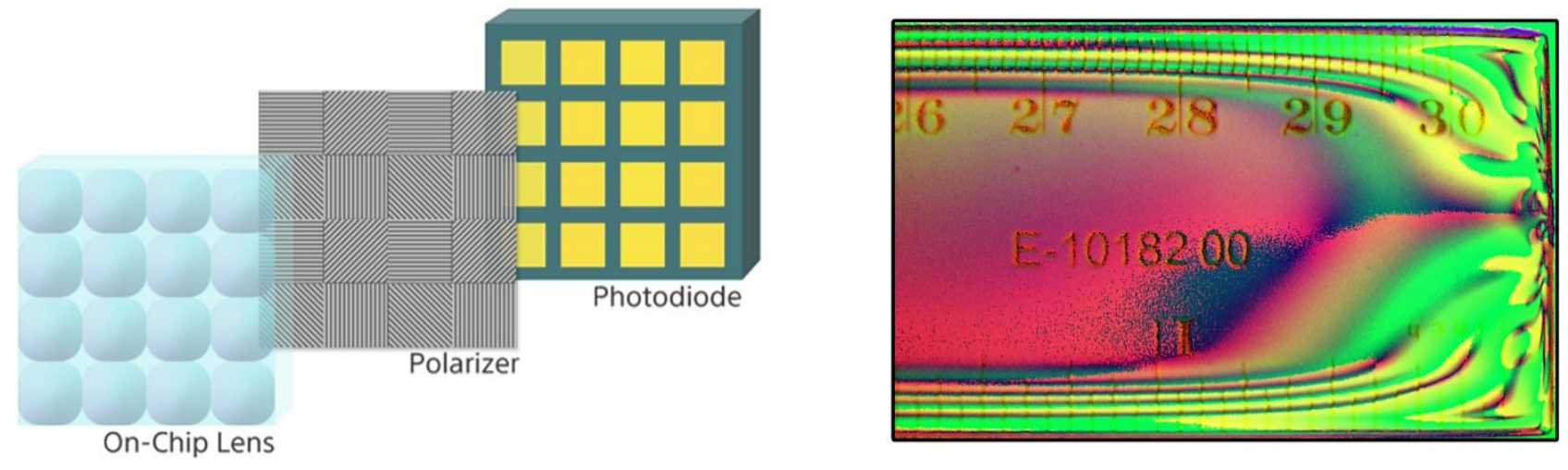

Figure 1. Left: Principal structure of the Sony IMX250MZR polarization image sensor [1]. Right: Internal stress of a transparent ruler made visible by false color presentation of the polarization. 
To describe the type of polarization, Stokes parameters are being used [2]:

$$
\begin{aligned}
& S_{0}=\left\langle E_{x}^{2}\right\rangle+\left\langle E_{y}^{2}\right\rangle, \\
& S_{1}=\left\langle E_{x}^{2}\right\rangle-\left\langle E_{y}^{2}\right\rangle, \\
& S_{2}=\left\langle 2 E_{x} E_{y} \cos \delta\right\rangle, \\
& S_{3}=\left\langle 2 E_{x} E_{y} \sin \delta\right\rangle .
\end{aligned}
$$

$E_{x}$ and $E_{y}$ are the time-averaged amplitudes of the electric wave vectors and $\delta$ describes their relative phase. The Stokes parameters are summarized in normalized Stokes vectors, which define the states of polarization [2]:

$$
S_{N}=\left(\begin{array}{l}
1 \\
0 \\
0 \\
0
\end{array}\right), \quad S_{x}=\left(\begin{array}{l}
1 \\
1 \\
0 \\
0
\end{array}\right), \quad S_{y}=\left(\begin{array}{c}
1 \\
-1 \\
0 \\
0
\end{array}\right), \quad S_{45^{\circ}}=\left(\begin{array}{l}
1 \\
0 \\
1 \\
0
\end{array}\right), \quad S_{-45^{\circ}}=\left(\begin{array}{c}
1 \\
0 \\
-1 \\
0
\end{array}\right), \quad S_{R}=\left(\begin{array}{l}
1 \\
0 \\
0 \\
1
\end{array}\right), \quad S_{L}=\left(\begin{array}{c}
1 \\
0 \\
0 \\
-1
\end{array}\right)
$$

The Stokes vector $S_{N}$ describes unpolarized light, the vectors $S_{x}, S_{y}, S_{45^{\circ}}$ and $S_{-45^{\circ}}$ describe linear polarized light in the xand y-plane and in the $45^{\circ}$ and $-45^{\circ}$ plane. The Stokes vectors $S_{R}$ and $S_{L}$ describe right- and left-circular polarized light.

Together with the appearance of the new FOC polarization sensors comes the need to characterize them with a uniform standard. The EMVA 1288 standard defines an accurate and reliable measurement procedure and guidelines for the presentation of the measurement results. Therefore, in this paper the sensor is characterized by the procedures of the EMVA 1288 standard, as far as they can be applied. The camera model used contains the IMX250MZR sensor and is a GigEVision industrial camera mvBlueCOUGAR-X manufactured by Matrix Vision GmbH.

\section{MEASUREMENT SETUP}

In order to measure the characteristic parameters of the FOC polarization camera, a setup containing a rotatable linear polarizer is used. Figure 2 shows an overview of the main components of the measurement setup. In this case a monochromator is used as a light source. The optical fiber of the monochromator is connected to a measurement tube, which contains a diffusor at the outlet of the fiber. The length of the measurement tube is constructed in accordance to the EMVA 1288 standard, therefore its output aperture is about f8 [3]. At the other end of the measurement tube the rotatable linear polarizer is placed. The linear polarizer used is made of PMMA and offers a transmission of $42 \%$ in the wavelength range $400 \mathrm{~nm}-700 \mathrm{~nm}$. The linear polarizer setup connects via C-Mount to the measurement tube and to the FOC polarization camera.

The rotatable linear polarizer is used to achieve a maximum transmission for one of the four channels of the IMX250MZR sensor. The maximum transmission is reached when the incident linear polarized light is orthogonal to the orientation of the wire grid. Therefore, the remaining three channels will receive smaller intensities of the incident light. In an ideal case, the channel with the grid polarizers being parallel to the orientation of the polarized light will receive $0 \%$ of the intensity of the incident light, for the remaining two channels with the grid orientation being in a $45^{\circ}$ angle to the orientation of the linear polarized light, the transmission is $50 \%$.

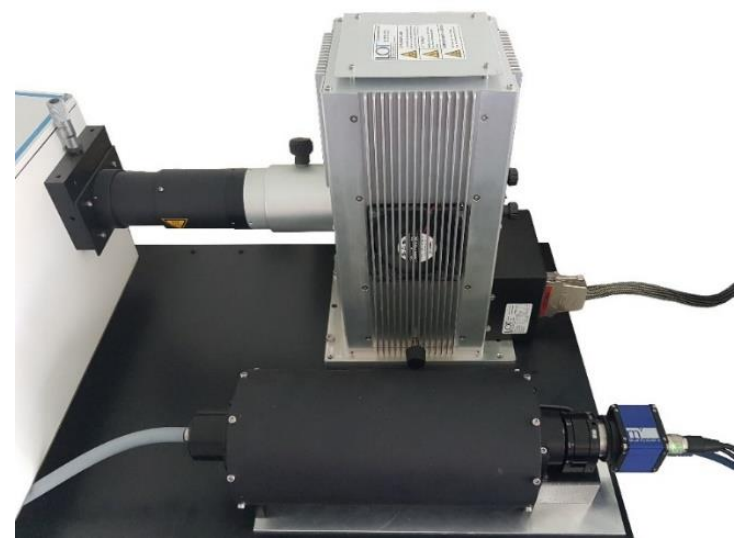

Figure 2. Overview of the measurement setup consisting of a monochromator as a light source, a measurement tube in accordance to the EMVA 1288 standard, a rotatable linear polarizer and the FOC polarization camera. 
For the characterization of a camera in accordance to the EMVA 1288 standard, it is necessary to measure the irradiance of the incident light hitting the sensor to calculate the number of incident photons. In order to achieve correct results, it is necessary to measure the irradiance after the light passed through the linear polarizer, which reduces the irradiance by its nature. Figure 3 shows the equipment used to measure the irradiance. Instead of the camera, an absolute calibrated photodiode is attached to the rotatable linear polarizer setup. The entrance window of the photodiode is placed in the correct equivalent distance to the image sensor, by an adapter manufactured in a rapid-prototyping process.

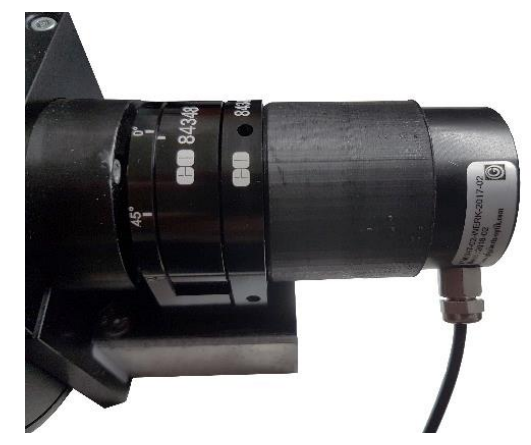

Figure 3. Measurement of the irradiance of the linear polarized incident light with a calibrated photodiode.

As a first approach to the characterization of FOC polarization sensors, this paper will focus on the camera parameter quantum efficiency and as a new parameter the extinction ratio of each wire grid channel. The quantum efficiency is measured for each channel individually. Therefore, the rotatable linear polarizer is positioned to allow maximum transmission for the observed channel. The correct alignment is checked before the measurement by analyzing a demosaiced live image of the camera. It is assumed, that the maximum transmission for the observed channel is reached when the both adjacent channels show the identical mean gray value (both should see $50 \%$ transmission). After the correct alignment is confirmed, a Signal Linearity Noise (SLN) measurement is performed for each channel, in accordance to the EMVA 1288 standard. The measurements were performed at a wavelength of $525 \mathrm{~nm}$ and for each measurement 30 picture pairs were taken.

\section{RESULTS AND DISCUSSION}

Figure 4 shows one of the four measured photon transfer curves. Taking measurement uncertainties into account, the quantum efficiency for each channel is the same, about $38 \%-39 \%$ at a wavelength of $525 \mathrm{~nm}$. Compared to the quantum efficiency of the Sony IMX250 image sensor (identical sensor without the FOC wire grids) with a quantum efficiency of $66 \%$ [4], this means a decrease of the quantum efficiency by $27 \%-28 \%$ caused by the wire grid polarizers. This is an expected result, caused by the transmission ratio of the FOC wire grids.

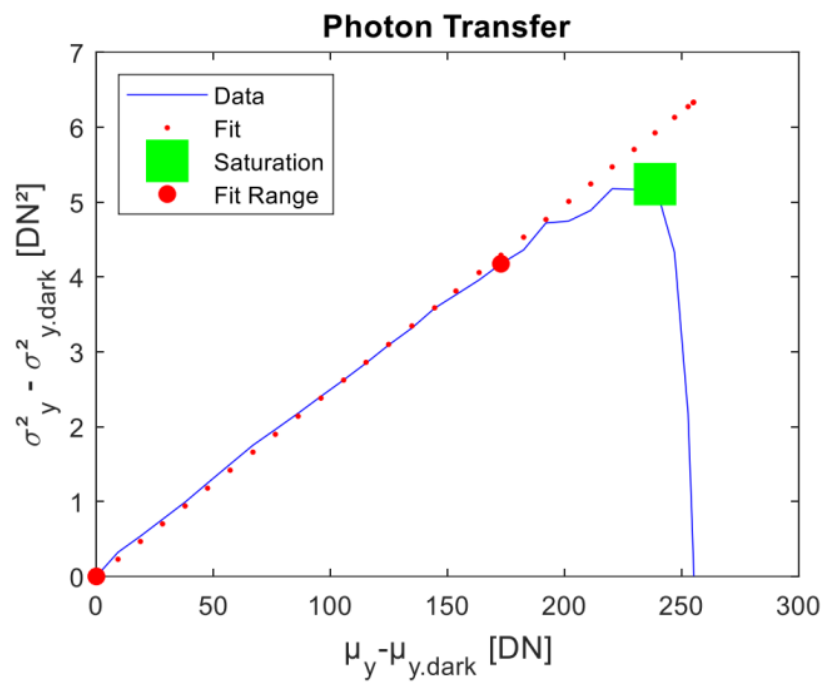

Figure 4. Measured photon transfer curve of the image sensor for the $90^{\circ}$ grid pixels @ $525 \mathrm{~nm}$ 
To extend the investigations, the quantum efficiency of the IMX250MZR Sensor was measured over the wavelength range of the rotatable linear polarizer $(400 \mathrm{~nm}-700 \mathrm{~nm})$ in accordance to the EMVA 1288 standard. The obtained results are shown in Figure 5.

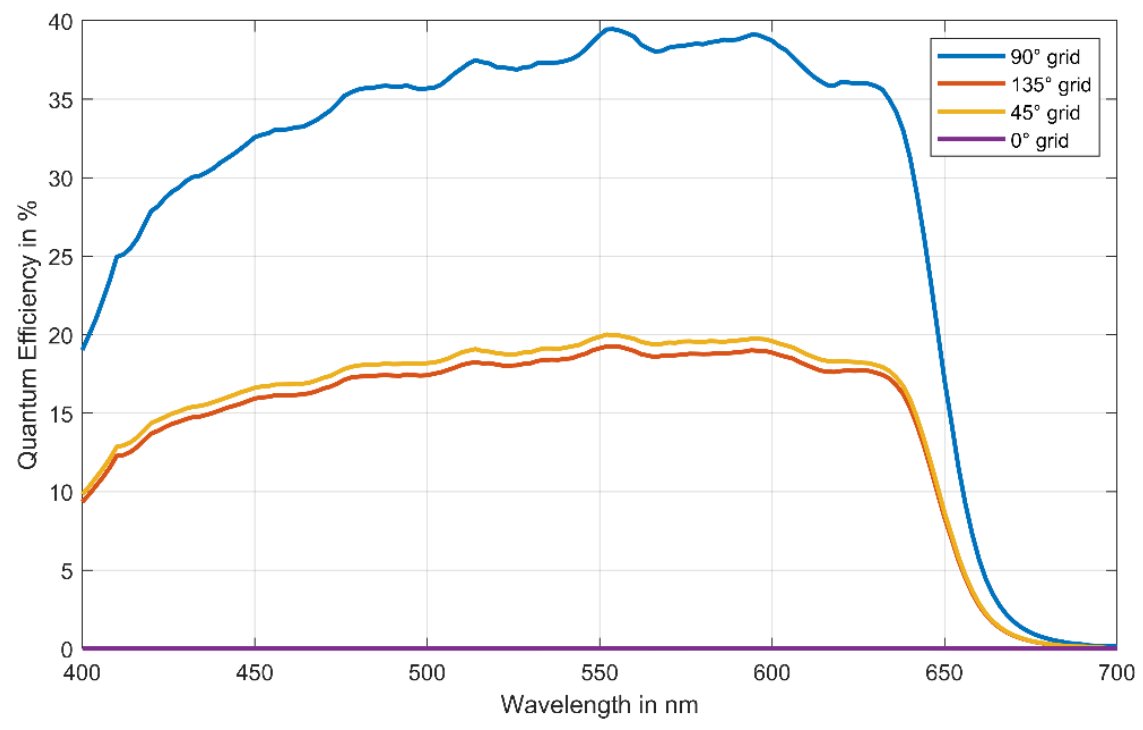

Figure 5. Measured Quantum Efficiency of all four channels with maximum transmission for the $90^{\circ}$ channel

The angle of the rotatable linear polarizer was adjusted to match a maximum transmission for the $90^{\circ}$ orientated wire grid polarizer channel. Therefore, this channel shows the highest quantum efficiency with a maximum of about $40 \%$ at $550 \mathrm{~nm}$ wavelength. The two adjacent channels $\left(135^{\circ}\right.$ and $\left.45^{\circ}\right)$ show approximately $50 \%$ of the quantum efficiency of the $90^{\circ}$ channel and the $0^{\circ}$ channel shows a quantum efficiency of $0 \%$. As pointed out in section 2, the quantum efficiency of the individual channels is as expected, when the linear polarization of the incident light is aligned with one of the wire grid angles. Little differences in the quantum efficiency of the $135^{\circ}$ and $45^{\circ}$ channel can be caused by a slight misalignment of the rotatable linear polarizer. The cutoff at about $650 \mathrm{~nm}$ can be explained by the operational wavelength range of the rotatable linear polarizer $(400 \mathrm{~nm}-700 \mathrm{~nm})$ and by the IR-cutoff filter of the FOC polarization camera.

When characterizing FOC polarization sensors, the extinction ratio $r_{e}$ must be taken into account as a new parameter. The extinction ratio is defined as:

$$
r_{e}=\frac{P_{1}}{P_{0}}
$$

where $P_{1}$ is in the case of a linear polarizer the intensity of light passing through the filter with maximum transmission and $P_{0}$ the intensity with minimum transmission. To calculate the extinction ratio for each wire grid channel, 10 images were taken for minimum and maximum transmission for each channel. The wavelength used is $525 \mathrm{~nm}$ and the images have a depth of 12 Bit. The average gray values were calculated for minimum and maximum transmission and then used to calculate the extinction ratio for every channel. The obtained results are shown in figure 6.

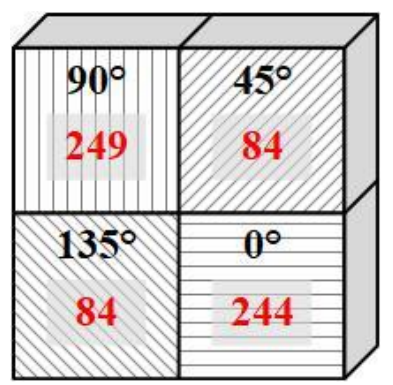

Figure 6. Determined extinction ratios (red) of the four different angles of the wire grid polarizers. 
It is noticeable that the extinction ratio for the channels which are not parallel to the axes of the image sensor $\left(45^{\circ}\right.$ and $135^{\circ}$ ) is smaller than the others. This could be an issue of the manufacturing process or due to the fact that the sensor that was characterized is a preproduction model. If the difference in the extinction ratio continues to occur in the actual production model and can be reproduced by other measurements, further investigations have to be done to find out the reason for this behavior.

\section{SUMMARY}

In summary characterizing a FOC polarization sensor does not differ much from characterizing conventional FOC sensors, like color sensors. In the case of color sensors, the EMVA 1288 standard clearly states to perform single measurements for each channel [3]. For FOC polarization sensors, instead of using different wavelengths to characterize each color channel, different angles of linear polarization are needed. Thus an adjustable linear polarizer is necessary in order to align the angle of polarization with the orientation of the on-chip wire grid polarizers to ensure that the maximum amount of light possible is hitting the polarization channel to be examined. Because only one polarization channel can reach maximum transmission at a time in the case of the IMX250MZR sensor, at least four different measurements at one operation point must be done to completely characterize the sensor at the chosen operation point.

A proposal to extend the EMVA 1288 standard to characterizing FOC polarization cameras is to add the channel-specific extinction ratio as an optional parameter. This can be easily achieved, because based on already performed individual SLN measurements for each channel, no additional measurements are needed, if all the demosaiced images are saved. This is because the minimum and maximum transmission occurs principle-based on two channels at a time.

\section{REFERENCES}

[1] Sony Semiconductor Solutions Corporation, https://www.sony-semicon.co.jp/products_en/IS/sensor5/index.html access at 03.06.2019

[2] Haferkorn, H., "Optik: physikalisch-technische Grundlagen und Anwendungen“, Wiley-VCH, Weinheim, 451 (2003)

[3] European Machine Vision Association 2016 EMVA Standard 1288 - Standard for Characterization of Image Sensors and Cameras (release 3.1) URL: www.emva.org

[4] LUCID Vision Labs Inc., "Going Polarized - Polarization Adds A New Perspective To The Imaging Industry”, 2018, https://www.jencam.de/WebRoot/Store/Shops/JenCam/5B68/2065/4A16/99A0/6188/AC1E/0A02/CA73/LUCID-

Going-Polarized-White-Paper.pdf 